\title{
Computation of ionization effect due to cosmic rays in polar middle atmosphere during GLE 70 on 13 December 2006
}

\author{
Alexander Mishev ${ }^{* \dagger}$ \\ ReSolve CoE University of Oulu, Finland. E-mail: alexander.misheveoulu.fi
}

Peter I.Y.I Velinov

Institute for Space Research and Technology, Bulgarian Academy of Sciences. E-mail: pvelinovabas.bg

\begin{abstract}
At recent one of the modern topics in solar-terrestrial physics is the study of the possible effect of solar variability, respectively cosmic rays variation on atmospheric physics and chemistry. An important feature in most of the proposed mechanisms and models is the key role of the induced by cosmic rays ionization. At recent is observed an apparent effect on minor constituents and aerosols over polar regions during major solar proton events. Therefore the ground level enhancement 70 on 13 December 2006 deserves a special attention. In this work we compute the ion production on 13 December 2006 on the basis on a previously applied model based on detailed Monte Carlo simulations of cosmic ray induced atmospheric cascade. The ion production rate during the event is considered as superposition of cosmic rays with galactic and solar origin. The time evolution of ion production is computed in a realistic manner. The spectral and angular characteristics of the solar protons are explicitly considered throughout the event as well their time evolution. The ionization effect during the event is computed at several altitudes above the sea level in a region with $R_{c} \leq 1 \mathrm{GV}$. The $24^{h}$ ionization effect is estimated in the region of the Pfotzer maximum.
\end{abstract}

The 34th International Cosmic Ray Conference,

30 July- 6 August, 2015

The Hague, The Netherlands

\footnotetext{
*Speaker.

$\dagger$ also at Institute for Nuclear Research and Nuclear Energy, Bulgarian Academy of Sciences.
} 


\section{Introduction}

Our planet is constantly hit by high-energy particles Galactic cosmic rays (GCRs). The majority are protons and $\alpha$-particles. They penetrate deep into the atmosphere producing large amount of secondaries. They are the main source of ionization in the troposphere and stratosphere [1, 2, 3 ]. The intensity of GCRs is modulated by the solar wind and follows the 11-year solar cycle. It responds to transient phenomena such as Forbush decreases [ [ of ionization is related to eruptive solar processes as solar flares and coronal mass ejections (CMEs), which produce solar energetic particles (SEPs) (see [5, 6] and references therein). Occasionally the SEPs initiate an atmospheric cascade leading to an enhancement of count rate of ground based neutron monitors (NMs), the so-called ground level enhancements (GLEs). They could significantly increase the ion production in the atmosphere, specifically in polar regions $[6,8,0,10,11,12$.

\section{Ion production rate and ionization effect during GLE 70}

In this work we assume a model similar to [13]. The detailed description of the model as well several features related to computations are given in [14, 15, 16,.

$$
Q\left(h, \lambda_{m}\right)=\frac{1}{E} \sum_{\text {ion }} \sum_{i} \int_{E}^{\infty} \int_{\Omega} D_{i}(E) \frac{\triangle E(h, E)}{\triangle h} \rho(h) d E d \Omega
$$

where $\triangle E$ is the deposited energy in an atmospheric layer $\triangle h, h$ is the air overburden (air mass) above a given altitude in the atmosphere expressed in $\mathrm{g} / \mathrm{cm}^{2}$ subsequently converted to altitude a.s.l., $D_{i}(E)$ is the differential cosmic ray spectrum for a given nuclei of primary $\mathrm{CR}, \rho$ is the atmospheric density in $\mathrm{g.cm}^{-3}, \lambda_{m}$ is the geomagnetic latitude, $E$ is the initial energy of the incoming primary nuclei on the top of the atmosphere, $\Omega$ is the geometry factor - a solid angle and $E_{\text {ion }}=35 \mathrm{eV}$ is the energy necessary for creation of an ion pair in air. The ion production rate during the GLE 70 is a superpose of the contribution of GCRs and SEPs. For the GCR spectrum we assume the force field approximation [17, 18, 19]. The SEPs rigidity spectra and angular distributions are considered according to reconstructions using NM data [20]. The computed ion production rate is presented in Fig.1a for $R_{c} \leq 1 \mathrm{GV}$ cut-off, accordingly Fig. $1 \mathrm{~b}$ for $R_{c} \leq 2 \mathrm{GV}$.

The derived ion production rate profiles allow us to estimate the time evolution of ion production (ion production [ion pairs $\mathrm{cm}^{-3}$ ] - integration of ion production rate over the phase of the event) throughout the event [21, 22] in a realistic atmospheric conditions [23, 24, 25]. The ion production at $12 \mathrm{~km}$ above the sea level (a.s.l.) during the initial phase Fig.2a, main phase Fig.2b and late phase Fig.2c are shown.

The corresponding ionization effect is shown in Fig.3. Accordingly the computed $24^{h}$ ionization effect in the region of the Pfotzer maximum is shown in Fig.4. As it was recently shown [22] the maximal ionization effect is observed on higher altitudes than the produced by the average of GCR. As example, during the event the ionization effect is $\approx 200 \%$ ( at altitude of $18 \mathrm{~km}$ a.s.l.) in the region of $60-180^{\circ} \mathrm{E}$ in the Southern hemisphere and diminish to about $150-160 \%$ at the altitude of $15 \mathrm{~km}$ a.s.l. The $24^{h}$ is about $20 \%$ in the region of Pfotzer maximum. In the troposphere the ionization effect sharply diminish. At altitudes below some $10 \mathrm{~km}$ a.s.l. the $24^{h}$ ionization effect is marginal or even negative in some regions due to significant Forbush decrease leading to reduce of a GCR flux. 


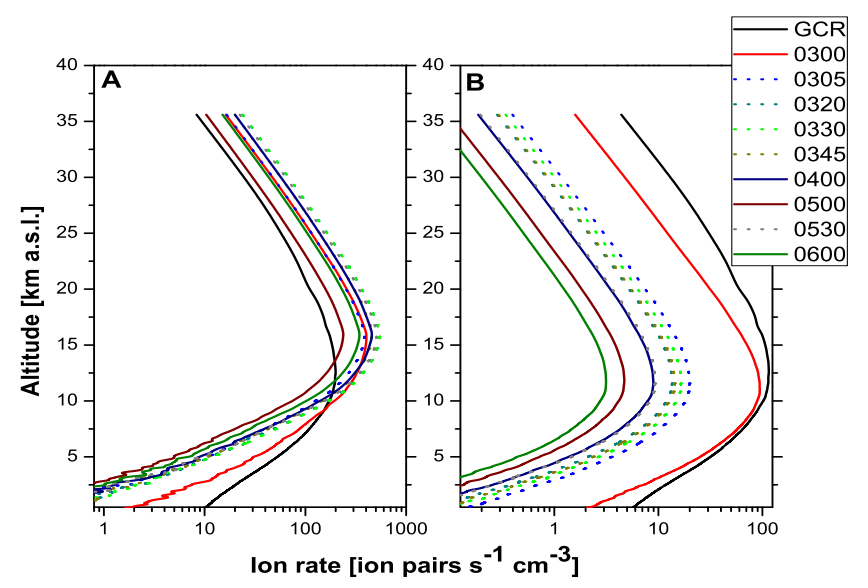

Figure 1: Ion production rate due to GCRs and GLE particles the GLE 70 on $13^{\text {th }}$ of December 2006. a) 1 GV rigidity cut-off; b) $2 \mathrm{GV}$ rigidity cut-off.).
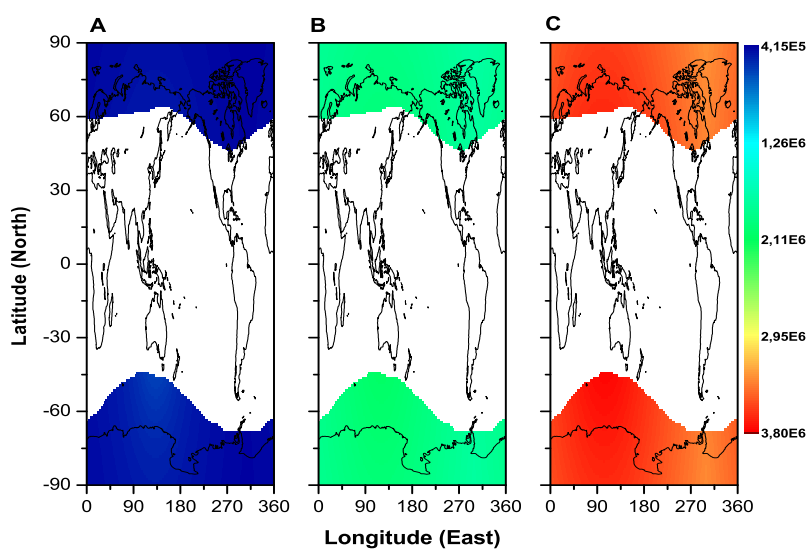

Figure 2: Ion production [ion pairs $\mathrm{cm}^{-3}$ ] at $12 \mathrm{~km}$ a.s.1. throughout GLE 70. a) 0300-0330 UT; b) 03300430 UT; c) 0430-0700 UT;

\section{Conclusion}

Here we present an assessment of ionization effect due to CRs of galactic and solar origin throughout the GLE 70. The ion production rate and ion production as well as the corresponding ionization effect varies throughout the event. This is due mainly on variation of apparent source position (movement to a North-West direction) as well as variation of spectral (SEP spectrum soften during the event) and angular characteristics (the pitch angle distribution broaden out). The ion production is maximal in the sub-polar and polar regions of Southern hemisphere, while it is minimal near to the anti-sunward direction. The ionization effect is important in a limited atmospheric region (range of altitudes a.s.l.), because of the rapidly falling and soft SEPs spectra. The anisotropy of SEPs considerably reflects on the magnitude of ionization effect in a given geographic region. This work is a contribution ot recent studies of this event [26]. 


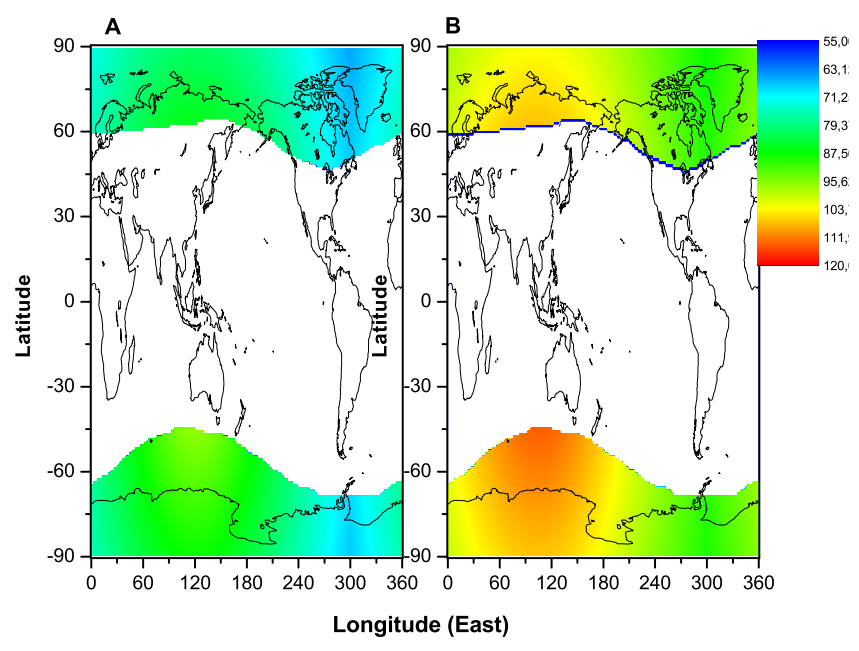

Figure 3: Ionization effect relative to average due to GCR during GLE 70. a) initial phase 0300-0330 UT; b) late phase $0430-0700 \mathrm{UT}$;

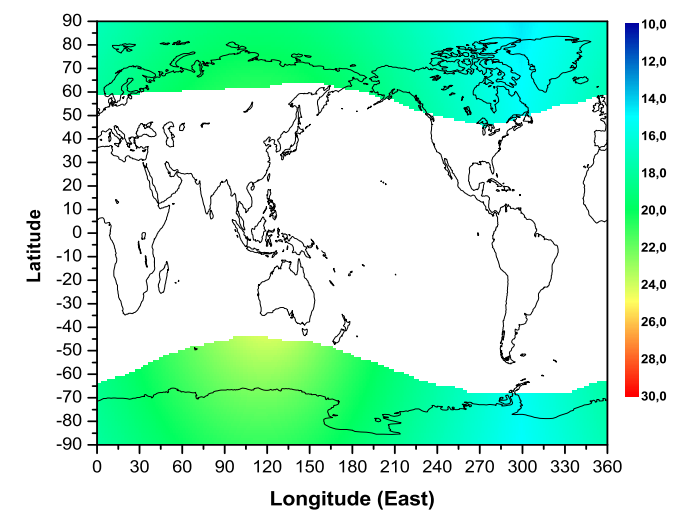

Figure 4: $24^{h}$ ionization effect in the region of the Pfotzer maximum

\section{Acknowledgements}

This work was supported by the Center of Excellence ReSoLVE (project No. 272157).

\section{References}

[1] G. A. Bazilevskaya, I. G. Usoskin, E. Flückiger, R. Harrison, L. Desorgher, B. Bütikofer, M. Krainev, V. Makhmutov, Y. Stozhkov, A. Svirzhevskaya, N. Svirzhevsky, and G. Kovaltsov, Cosmic ray induced ion production in the atmosphere, Space Science Reviews 137 (2008) 149-173.

[2] I. G. Usoskin, L. Desorgher, P. Velinov, M. Storini, E. Flückiger, R. Bütikofer, and G. Kovaltsov, Ionization of the earth's atmosphere by solar and galactic cosmic rays, Acta Geophysica 57 (2009), no. $188-101$. 
[3] P. Velinov, S. Asenovski, K. Kudela, J. Lastovička, L. Mateev, A. Mishev, and P. Tonev, Impact of cosmic rays and solar energetic particles on the earth's ionosphere and atmosphere, Journal of Space Weather and Space Climate 3 (2013).

[4] S. Forbush, On the effects in cosmic-ray intensity observed during the recent magnetic storm, Physical Review 51 (1937), no. 121108.

[5] M. Aschwanden, Gev particle acceleration in solar flares and ground level enhancement (gle) events, Space Science Reviews 171 (2012), no. 1-4 3-21.

[6] L. Miroshnichenko, Solar Cosmic Rays: Fundamentals and Applications. Springer, Dordrecht, 2014.

[7] A. Mishev, P. Velinov, L. Mateev, and Y. Tassev, Ionization effect of solar protons in the earth atmosphere - case study of the 20 january 2005 sep event, Advances of Space Research 48 (2011), no. 7 1232-1237.

[8] A. Mishev, P. Velinov, and L. Mateev, Ion production rate profiles in the atmosphere due to solar energetic particles on 28 october 2003 obtained with corsika 6.52 simulations, Comptes Rendus de L'Academie Bulgare des Sciences 64 (2011), no. 6 859-866.

[9] I. G. Usoskin, G. Kovaltsov, I. Mironova, A. Tylka, and W. Dietrich, Ionization effect of solar particle gle events in low and middle atmosphere, Atmospheric Chemistry and Physics 11 (2011) 1979-1988.

[10] A. Mishev, P. Velinov, L. Mateev, and Y. Tassev, Ionization effect of nuclei with solar and galactic origin in the earth atmosphere during gle 69 on 20 january 2005, Journal of Atmospheric and Solar-Terrestrial Physics 89 (2013), no. 1 1-7.

[11] I. Mironova, I. Usoskin, G. Kovaltsov, and S. Petelina, Possible effect of extreme solar energetic particle event of 20 january 2005 on polar stratospheric aerosols: Direct observational evidence, Atmospheric Chemistry and Physics 12 (2012), no. 2 769-778.

[12] P. Velinov and A. Mishev, Comparison of ionization effect in the atmosphere of the earth due to gle 65 and gle 69, Journal of Physics: Conference Series 409 (2013), no. 1.

[13] I. Usoskin and G. Kovaltsov, Cosmic ray induced ionization in the atmosphere: Full modeling and practical applications, Journal of Geophysical Research 111 (2006), no. D21206.

[14] P. Velinov, A. Mishev, and L. Mateev, Model for induced ionization by galactic cosmic rays in the earth atmosphere and ionosphere, Advances in Space Research 44 (2009), no. 9 1002-1007.

[15] A. Mishev and P. I. Velinov, Normalized ionization yield function for various nuclei obtained with full monte carlo simulations, Advances of Space Research 48 (2011), no. 1 19-24.

[16] A. Mishev and P. Velinov, Influence of hadron and atmospheric models on computation of cosmic ray ionization in the atmosphere-extension to heavy nuclei, Journal of Atmospheric and Solar-Terrestrial Physics 120 (2014) 111-120.

[17] L. Gleeson and W. Axford, Solar modulation of galactic cosmic rays, Astrophysical Journal 154 (1968) 1011-1026.

[18] R. Caballero-Lopez and H. Moraal, Limitations of the force field equation to describe cosmic ray modulation, Journal of Geophysical Research 109 (2004) A01101.

[19] I. Usoskin, K. Alanko-Huotari, G. Kovaltsov, and K. Mursula, Heliospheric modulation of cosmic rays: Monthly reconstruction for 1951-2004,, Journal of Geophysical Research 110 (2005), no. A12108. 
[20] E. Vashenyuk, Y. Balabin, B. Gvozdevsky, and L. Schur, Characteristics of relativistic solar cosmic rays during the event of december 13, 2006, Geomagnetism and Aeronomy 48 (2008), no. 2 149-153.

[21] A. Mishev and P. Velinov, A maverick gle 70 in solar minimum. calculations of enhanced ionization in the atmosphere due to relativistic solar energetic particles, Comptes Rendus de L'Academie Bulgare des Sciences 66 (2013), no. 10 1457-1462.

[22] A. Mishev and P. Velinov, Time evolution of ionization effect due to cosmic rays in terrestrial atmosphere during gle 70, Journal of Atmospheric and Solar-Terrestrial Physics 129 (2015) 78-86.

[23] A. Mishev and P. Velinov, Effects of atmospheric profile variations on yield ionization function y in the atmosphere, Comptes Rendus de L'Academie Bulgare des Sciences 61 (2008), no. 5 639-644.

[24] A. Mishev, P. Velinov, V. Yanke, and E. Eroshenko, Effects of different atmospheric profiles on ionization in the earth atmosphere, in Proc. of 31th ICRC Lodz, Poland, 7 -15 July 2009, 2009.

[25] A. Mishev and P. Velinov, The effect of model assumptions on computations of cosmic ray induced ionization in the atmosphere, Journal of Atmospheric and Solar-Terrestrial Physics 72 (2010), no. 5-6 476-481.

[26] V. Žigman, K. Kudela, and D. Grubor, Response of the earth's lower ionosphere to the ground level enhancement event of december 13, 2006, Advances in Space Research 53 (2014), no. 5 763-775. 